\title{
Characterisation of an oxidative response inhibitor produced by Streptococcus pneumoniae
}

\author{
F E Perry, C J Elson, T J Mitchell, P W Andrew, J R Catterall
}

\begin{abstract}
Background - Pneumonia caused by infection with Streptococcus pneumoniae is still a major clinical problem. Reactive oxygen species contribute to the killing of these bacteria by polymorphonuclear leucocytes (PMNs). Defence mechanisms of Str pneumoniae which counter reactive oxygen species are characterised.

Methods - PMNs were stimulated with phorbol myristate acetate (PMA) in the presence and absence of Str pneumoniae and supernatants from them, and superoxide $\left(\mathrm{O}_{2}^{-}\right)$production was measured by the reduction of ferricytochrome $c$.
\end{abstract}

Results - Streptococcus pneumoniae, but not Klebsiella pneumoniae or Staphylococcus aureus, inhibited PMA stimulated superoxide production by PMNs. Washed PMNs which had been preincubated with Str pneumoniae autolysis phase supernatants also exhibited depressed $\mathrm{H}_{2} \mathrm{O}_{2}$ production in response to $P M A$. The inhibitory activity was not attributable to non-specific cytotoxicity as assessed by release of the cytoplasmic enzyme lactate dehydrogenase, nor did the supernatants inhibit PMA stimulated degranulation of PMNs. Fractionation of the autolysis phase supernatants revealed inhibitory activity in both the fractions greater than and less than $10 \mathrm{kD}$. Like pneumolysin the inhibitory activity was heat sensitive. However, both a parent and pneumolysin negative mutant Str pneumoniae, and autolysis phase supernatants from them, inhibited PMN superoxide production. Antisera to pneumolysin failed to abrogate the inhibitory effect of intact Str pneumoniae or autolysis phase supernatants from types 1 or 14 Str pneumoniae.

Conclusions - The inhibitory effect of Str pneumoniae on the respiratory burst of PMNs is not shared by two other common lung pathogens. The existence of a novel inhibitor of the PMN respiratory burst, distinct from pneumolysin, has been demonstrated. The inhibitor is specific for the respiratory burst and is active both in the logarithmic phase of growth and during autolysis.

(Thorax 1994;49:676-683)

Despite antibiotics, infections caused by Streptococcus pneumoniae continue to cause a high morbidity and mortality. Pneumococcal pneumonia, for example, is the most common form of community acquired pneumonia in hospital ${ }^{1}$ with an overall mortality of $5-13 \%$, whilst meningitis caused by Str pneumoniae carries a mortality of $30 \% .^{3}$ Furthermore, the worldwide increase in penicillin resistance and multiple antibiotic resistance among pneumococci, ${ }^{4-7}$ and the limited use of pneumococcal vaccine ${ }^{8}$ suggest that pneumococcal infection may cause increasing morbidity and mortality in future years.

In the host's defence against Str pneumoniae killing by phagocytes has a key role. ${ }^{910}$ The mechanism of killing is incompletely understood, but there is evidence that, as with the killing of many other microorganisms, ${ }^{11-13}$ the release of oxygen species by phagocytes is important. For example, Str pneumoniae is killed by reactive oxygen species; $;^{14}$ polymorphonuclear leucocytes (PMNs) incubated anaerobically have a diminished capacity to kill Str pneumoniae, ${ }^{16}$ and Str pneumoniae can stimulate PMNs to release superoxide under certain conditions. ${ }^{1718}$ Recent studies in our laboratories, however, have shown that Str pneumoniae in suspension are able to interfere with the oxidative burst of PMNs. ${ }^{19}$ They inhibit both spontaneous superoxide production by PMNs and the respiratory burst stimulated by phorbol myristate acetate (PMA) The effect is associated with no change in viability of the PMNs (assessed by trypan blue exclusion), and is evident within 15 minutes of adding the organism. The inhibitory activity is exhibited by intact organisms in the logarithmic phase of growth and is released on autolysis. Inhibition is dependent on both the dose and viability of the bacteria and was demonstrated for two different serotypes which are among the major causative organisms of serious pneumococcal infections. Given the central role of phagocytes in the host's defence against Str pneumoniae, and the importance of reactive oxygen species in the killing of bacteria by phagocytes, this inhibitory activity may be an important defence mechanism of Str pneumoniae.

The purpose of the current work was (1) to determine whether the inhibitory activity was specific to Str pneumoniae and to the respiratory burst, and (2) to characterise the activity further. Since pneumolysin (a toxin released on autolysis of Str pneumoniae) also has been shown to inhibit the respiratory burst of PMNs, ${ }^{20}$ we were particularly interested to determine whether the inhibitory activity that we have observed could be accounted for by pneumolysin. Two approaches were taken: the ability of a pneumolysin negative strain of Str pneumoniae to inhibit the respiratory burst was investigated and the effect of antipneumolysin serum on the inhibitory activity of Str pneumoniae was assessed. 
Methods

STREPTOCOCCUS PNEUMONIAE

Encapsulated types 1 and 14 Str pneumoniae were obtained from patients with bacteraemic pneumococcal pneumonia. The pneumolysin negative Str pneumoniae was a mutant of serotype 2 which had been constructed previously by disrupting the gene encoding pneumolysin by insertion-duplication mutagenesis. ${ }^{21}$ The bacteria were stored in $1 \mathrm{ml}$ aliquots at $-70^{\circ} \mathrm{C}$. Before use, thawed Str pneumoniae was grown in Todd-Hewitt broth with $10 \%$ (v/v) new born calf serum for 3-4 hours so that the organisms were in the logarithmic phase of growth (log phase organisms). The bacteria were collected by centrifugation at $2200 \mathrm{~g}$ for 10 minutes, washed twice, resuspended in phosphate buffered saline (PBS), and adjusted to $10 \times 10^{7} / \mathrm{ml}$. The total bacterial count was determined with a Helber chamber and the viable count assessed by serial dilution and plating onto blood agar, using the method of Miles and Misra. ${ }^{22}$ In some experiments Str pneumoniae was used which had been grown for 18-24 hours, by which time the organisms had undergone autolysis (autolysis phase).

\section{KLEBSIELLA PNEUMONIAE AND \\ STAPHYLOCOCCUS AUREUS}

Clinical isolates of $K$ pneumoniae and Staph aureus were obtained from the Bacteriology Laboratory, Bristol Royal Infirmary. They were stored and prepared as for Str pneumoniae, using nutrient broth as the culture medium.

\section{PREPARATION OF CULTURE SUPERNATANTS}

The supernatant from the first centrifugation of the bacterial culture was retained for use in some experiments. As appropriate to the experiment, the supernatants were treated in the following ways.

\section{Filtration}

Sterilisation of bacterial culture supernatants was achieved by filtration through a $0.2 \mu \mathrm{m}$ filter (Millipore).

\section{Heat treatment}

Culture supernatants were heat treated by incubation in a water bath at $60^{\circ} \mathrm{C}$ for 30 minutes.

\footnotetext{
Dialysis

Dialysis of supernatants $(3-5 \mathrm{ml})$ was carried out in Visking tubing with a molecular weight cut off of $10 \mathrm{kD}$ against $500 \mathrm{ml}$ Todd-Hewitt broth overnight at $4^{\circ} \mathrm{C}$ and again for four hours at room temperature with a magnetic stirrer against $500 \mathrm{ml}$ fresh Todd-Hewitt broth.
}

\section{Fractionation}

Culture supernatants were separated into high and low molecular weight fractions by centrifugation in Millipore Ultrafree-MC filters with $10 \mathrm{kD}$ cut off membranes at $1110 \mathrm{~g}$ for three hours. The $>10 \mathrm{kD}$ material retained in the filter cup was resuspended to the original volume in Todd-Hewitt broth.

\section{POLYMORPHONUCLEAR LEUCOCYTES}

Peripheral blood was collected from healthy volunteers attending the outpatients department (mainly for elective surgery) at Bristol Royal Infirmary. Blood was obtained by venepuncture and collected in $3.8 \%(\mathrm{w} / \mathrm{v})$ sodium citrate. In each experiment blood from one donor was used. PMNs were isolated by dextran sedimentation followed by centrifugation through Percoll (Sigma) using a modification of the method of Dooley et al. ${ }^{23}$ The cells obtained were washed and resuspended in PBS with $4 \%$ citrate at $4 \times 10^{6} / \mathrm{ml}$. Ethical approval for obtaining blood from healthy volunteers was provided by the ethical committee of the Bristol and Weston Health Authority.

\section{REAGENTS}

Ferricytochrome c (type III), superoxide dismutase (SOD, type I, $300 \mathrm{U} / \mathrm{mg}$ protein), phorbol myristate acetate (PMA), phenol red, horseradish peroxidase, cytochalasin b $(1 \mathrm{mg} / \mathrm{ml}$ in DMSO) and Triton X-100 were obtained from Sigma (Poole, Dorset, UK). The PMA was dissolved in DMSO at $1 \mathrm{mg} / \mathrm{ml}$ and stored in $10 \mu \mathrm{l}$ aliquots at $-20^{\circ} \mathrm{C}$. The cytochrome $c$ and SOD were dissolved in PBS citrate, either made fresh on the day of the experiment or stored at $-20^{\circ} \mathrm{C}$.

\section{SUPEROXIDE DETECTION}

The amount of superoxide $\left(\mathrm{O}_{2}^{-}\right)$released by PMNs either spontaneously or in response to PMA or Str pneumoniae was measured by the microassay of Pick and $\mathrm{Mizel}^{24}$ which uses SOD inhibitable reduction of ferricytochrome c. The reaction mixture $(100 \mu \mathrm{l})$ consisted of $160 \mu \mathrm{mol} / 1$ cytochrome c, $1 \times 10^{5}$ PMNs, and the appropriate stimulus (Str pneumoniae or $1 \mu \mathrm{g} / \mathrm{ml}$ PMA), with or without $300 \mathrm{U} / \mathrm{ml}$ SOD. Six or eight replicates of each condition were set up in vertical rows of a 96-well flat bottomed tissue culture plate (Nunc, Denmark). The plate was covered and placed in a humidified incubator gassed with $95 \%$ air and $5 \%$ carbon dioxide for 90 minutes. The plate was then read on a Multiskan MCC/340 plate reader (Titertek) fitted with a $550 \mathrm{~nm}$ wavelength interference filter. The amount of superoxide produced per well was calculated from the formula ${ }^{24}$ :

$$
\begin{aligned}
& \text { nmoles } \mathrm{O}_{2}^{-} \text {per well } \\
& =\frac{(\text { absorbance at } 550 \mathrm{~nm} \times 100)}{6.51}
\end{aligned}
$$

HYDROGEN PEROXIDE DETECTION

Hydrogen peroxide production by PMNs was measured by hydrogen peroxide dependent reduction of phenol red by horseradish peroxi- 
dase. Phenol red solution was prepared using $4.64 \mathrm{ml}$ Hank's balanced salt solution $+4 \%$ $(\mathrm{w} / \mathrm{v})$ bovine serum albumin: $0.09 \mathrm{ml} 28 \mathrm{mmol} / 1$ phenol red: $0.07 \mathrm{ml} 12 \mathrm{mg} / \mathrm{ml}$ horseradish peroxidase. Phenol red solution, $0.5 \mathrm{ml}$, was combined with $10^{6}$ PMNs and PMA in replicate wells of a 24-well plate (final volume $1 \mathrm{ml}$ ). The mixtures were incubated for 30 minutes at $37^{\circ} \mathrm{C}$ and the reaction terminated by the addition of $0.01 \mathrm{ml} 1 \mathrm{~N} \mathrm{NaOH}$. The plates were centrifuged at $110 \mathrm{~g}$ for five minutes to remove the PMNs and the supernatants transferred to $1 \mathrm{ml}$ cuvettes. The absorbance of the solutions was measured at $610 \mathrm{~nm}$ and the data converted to nanomoles of hydrogen peroxide by means of a standard curve which was established for each experiment.

\section{DEGRANULATION ASSAY}

Neutrophil degranulation was measured by assaying for the azurophil enzyme myeloperoxidase. Neutrophils were pretreated with cytochalasin b $(20 \mu \mathrm{g} / \mathrm{ml})$ for 10 minutes at room temperature, then incubated (at $10^{6} / \mathrm{ml}$ ), either alone or with PMA $(1 \mu \mathrm{g} / \mathrm{ml})$, or $S t r$ pneumoniae autolysis phase supernatants (final concentration $1 / 4 \mathrm{v} / \mathrm{v}$ ), or both, for 60 minutes at $37^{\circ} \mathrm{C}$. Myeloperoxidase activity in the PMN supernatants was measured using an adaptation of the method of Suzuki et al ${ }^{25}$ utilising $3,3^{\prime}, 5,5^{\prime}$ tetramethyl benzidine (TMB). Triplicate aliquots $(150 \mu \mathrm{l})$ of the supernatant to be assayed were added to the assay substrate (20 mmol/l TMB $(50 \mu \mathrm{l}), 0.03 \%$ hydrogen peroxide $(\mathrm{v} / \mathrm{v}, 20 \mu \mathrm{l}), 0.01 \mathrm{mmol} / \mathrm{l}$ sodium acetate buffer pH 5.2 $(50 \mu \mathrm{l})$ and $150 \mu \mathrm{l}$ PBS $)$ and incubated for exactly five minutes. The reaction was terminated by placing the reagents on ice. PBS, $1 \mathrm{ml}$, was added and the absorbance at $655 \mathrm{~nm}$ measured against a substrate blank in a Shimadzu spectrophotometer. Enzyme release was expressed as a percentage of the maximum releasable. This was determined by lysing PMNs with $0.05 \%$ Triton X-100 for two minutes.

\section{LACTATE DEHYDROGENASE ASSAY}

Release of the cytoplasmic enzyme lactate dehydrogenase (LDH) was used as a marker of cell death. The amount of $\mathrm{LDH}$ in the supernatants of PMNs $\left(10^{6} / \mathrm{ml}\right)$ incubated either alone, with Str pneumoniae autolysis phase supernatants (final concentration $1 / 4 \mathrm{v} / \mathrm{v}$ ), or with $0.05 \%$ Triton $\mathrm{X}-100$ for 90 minutes at $37^{\circ} \mathrm{C}$ was assayed using a standard kit (Sigma). Spectrophotometric absorbance data were converted to Berga-Broida (BB) units of LDH activity by means of a standard curve.

PNEUMOLYSIN AND ANTIPNEUMOLYSIN SERUM Recombinant pneumolysin, ${ }^{2627}$ normal rabbit serum, and antipneumolysin rabbit serum were prepared as described elsewhere. ${ }^{27}$

SHEEP RED BLOOD CELL HAEMOLYSIS ASSAY In order to measure the amount of pneumolysin present in Str pneumoniae log phase cultures and autolysis phase supernatants, an adaptation of the method of Steinfort et $a l^{28}$ was used.
Doubling dilutions of recombinant pneumolysin, Str pneumoniae culture, or autolysis supernatants were prepared and incubated with or without 1:100 antipneumolysin serum for one hour at room temperature. Fresh sheep red blood cells $(2 \% \mathrm{v} / \mathrm{v})$ were added and the plate incubated for 30 minutes at $37^{\circ} \mathrm{C}$. The plate was then centrifuged for two minutes at $110 \mathrm{~g}$ and $50 \mu \mathrm{l}$ of the supernatant was transferred to a 96well flat bottomed plate. Absorbance was read at $550 \mathrm{~nm}$ and the values obtained expressed as a percentage of total haemolysis measured in sheep red blood cells lysed with distilled water. The $\mathrm{pH}$ of autolysis supernatants was adjusted to $7 \cdot 0-7 \cdot 2$ for use in the haemolysis assays since preliminary experiments had shown that non$\mathrm{pH}$ adjusted supernatants which were 5.3-6.4 had some lytic activity when mixed 1:1 with sheep red blood cells $(27.8 \%(6.3 \%)$ haemolysis, $n=10$ ), which in only five samples was partially reduced by antipneumolysin rabbit serum $(17.8 \%(4.8 \%)$ haemolysis $)$ and which was lost $(2 \cdot 7 \%(2 \cdot 1 \%)$ haemolysis, $\mathrm{n}=10$ ) when the supernatants were first diluted with an equal volume of PBS to give the same concentration as used in the superoxide assay. These results suggested that the haemolysis caused by non-pH adjusted supernatants was due to their acidity.

\section{STATISTICAL ANALYSIS}

Each experiment had either six or eight replicates per condition. For comparisons between experiments the mean value of the replicates was used. Results are expressed as the mean (SD) of the combined experiments. Unless otherwise stated, the paired $t$ test was used to assess differences between means, $p<0.05$ being considered significant.

\section{Results}

OXIDATIVE RESPONSES OF PMNS TO DIFFERENT RESPIRATORY PATHOGENS

The results of incubating PMNs with either washed log phase Str pneumoniae (type 1), Klebsiella pneumoniae, or Staphylococcus aureus at a PMN:bacteria ratio of $1: 20$ in the presence of $10 \%$ pooled human serum are shown in fig 1. Streptococcus pneumoniae reduced the amount of spontaneous superoxide detected as previously reported. ${ }^{19}$ By contrast, in each of five experiments both $K$ pneumoniae and Staph aurens stimulated release of superoxide. It should be noted that the experiments with $S t r$ pneumoniae were performed at different times from those with the other organisms, accounting for the difference in spontaneous superoxide release. Further experiments were set up to determine whether $K$ pneumoniae and Staph aureus are able to inhibit PMA stimulated superoxide production. The results are presented in fig 2 and show that, in sharp contrast to Str pneumoniae, neither $K$ pneumoniae nor Staph aureus interfered with the respiratory burst of PMNs.

\section{SPECIFICITY OF THE INHIBITOR}

We have previously shown that the inhibitory activity of Str pneumoniae is not the result of 


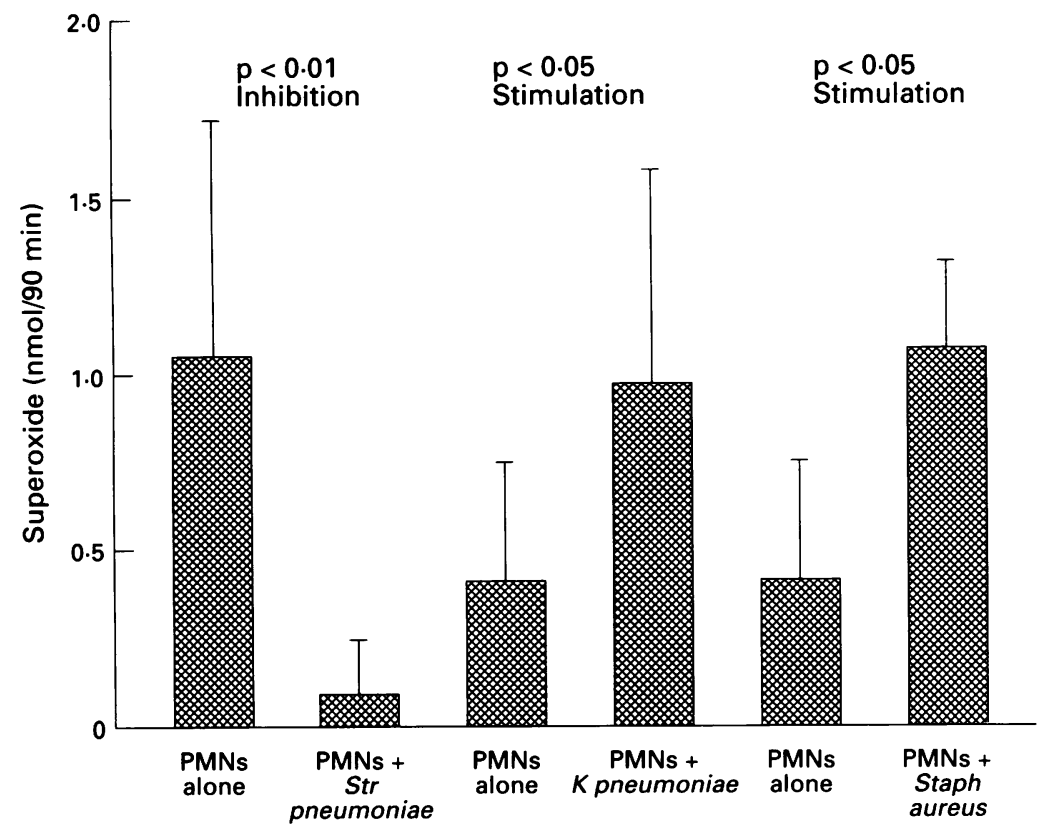

Figure 1 Oxidative response of polymorphonuclear leucocytes (PMNs) to log phase Str pneumoniae, $K$ pneumoniae, and Staph aureus in the presence of $10 \%$ pooled human serum. Results are mean (SD) of five experiments.

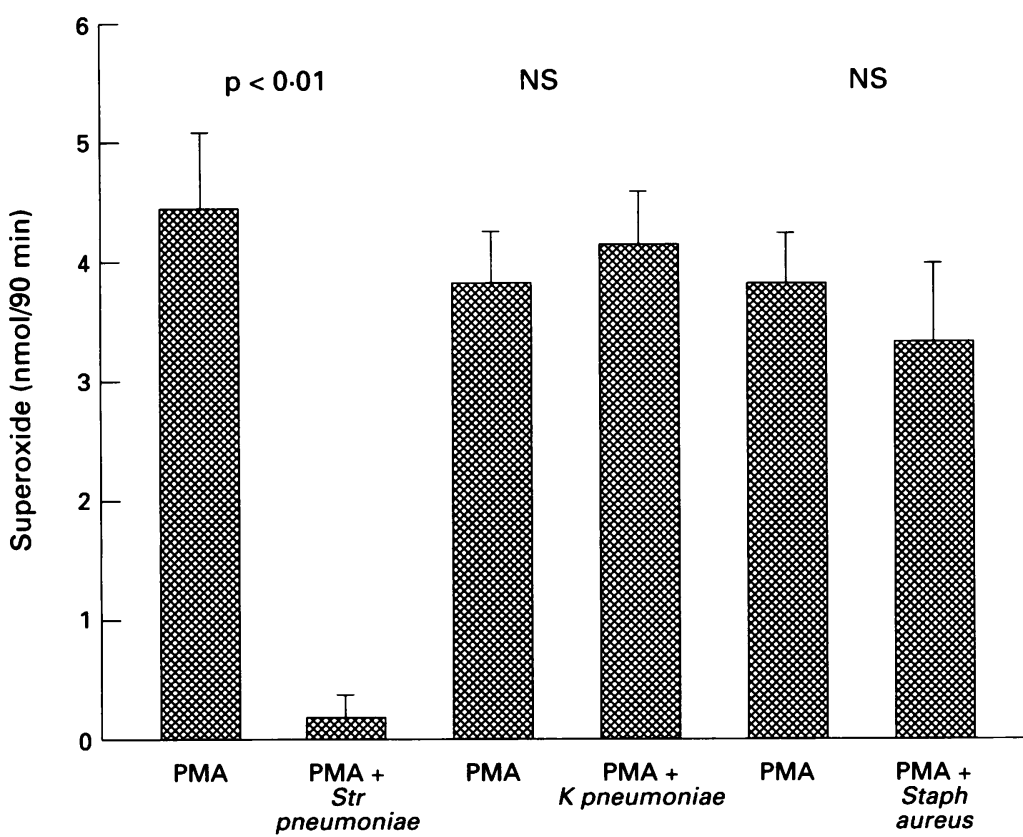

Figure 2 Effect of log phase Str pneumoniae, $K$ pneumoniae, and Staph aureus on phorbol myristate acetate (PMA) stimulated superoxide production by polymorphonuclear leucocytes (PMNs). Results are mean ( $S D)$ of five experiments.
To assess whether Str pneumoniae autolysis phase supernatants inhibited or affected other PMN functions their effect on PMN degranulation was investigated. The results are shown in table 1 and demonstrate that the supernatants did not inhibit PMA stimulated myeloperoxidase release but, rather, significantly enhanced it. This finding raised the possibility that the supernatants were stimulating PMN degranulation. PMNs incubated with autolysis phase supernatants, however, did not release significantly more myeloperoxidase than PMNs incubated alone.

\section{INHIBITION OF HYDROGEN PEROXIDE}

PRODUCTION BY STR PNEUMONIAE

To reinforce the conclusion that Str pneumoniae inhibited the respiratory burst of PMNs, the release of a second product, hydrogen peroxide, was measured. PMN suspensions were preincubated with either Todd-Hewitt broth or $\mathrm{pH}$ adjusted ( $\mathrm{pH} 7 \cdot 2$ ) autolysis phase supernatants (at a volume:volume ratio of 1 ) for one hour at $37^{\circ} \mathrm{C}$ and washed twice in PBS before stimulation with PMA. After 30 minutes hydrogen peroxide production was measured. PMNs preincubated with autolysis phase supernatants generated significantly less hydrogen peroxide in response to PMA than control PMNs preincubated with Todd-Hewitt broth: PMNs + Todd-Hewitt broth 28.59 $(2 \cdot 11) \mathrm{nmol}$ hydrogen peroxide/30 minutes, PMNs + autolysis phase supernatant 17.02 $(8.02) \mathrm{nmol}$ hydrogen peroxide/30 minutes, $\mathrm{n}=3, \mathrm{p}<0.05$. The viability of the PMNs in these experiments was $96-98 \%$ as judged by trypan blue exclusion.

CHARACTERISATION OF THE RESPIRATORY BURST INHIBITOR PRODUCED BY STR PNEUMONIAE We have previously reported ${ }^{19}$ that the inhibitory activity of Str pneumoniae is associated with washed organisms in the logarithmic phase of growth and is not released into the log phase supernatant, but that it is released into the culture supernatant during autolysis. As a first step in characterising the inhibitory activity its heat sensitivity was investigated. In four experiments autolysis phase supernatants heated for 30 minutes at $60^{\circ} \mathrm{C}$ lost their ability to inhibit PMA stimulated superoxide production: PMNs + PMA + Todd-Hewitt broth (control) $4.52(0.64) \mathrm{nmol} \mathrm{O} 2^{-} / 10^{5} \mathrm{PMNs} / 90$ minutes, PMNs + PMA + untreated autolysis supernatant $1.51(1.11) \mathrm{nmol} \mathrm{O}_{2}^{-} / 10^{5} \mathrm{PMNs} /$ 90 minutes $(\mathrm{p}<0.01), \mathrm{PMNs}+\mathrm{PMA}+$ heat non-specific toxicity towards PMNs as treated autolysis supernatant $4.33(0.89) \mathrm{nmol}$ assessed by trypan blue exclusion. ${ }^{19}$ As an $\mathrm{O}_{2}^{-} / 10^{5} \mathrm{PMNs} / 90$ minutes. Filtered supernaadditional test of PMN viability, the more tants from the autolysis phase of growth were sensitive method of lactate dehydrogenase re- then dialysed (exclusion pore size 10000 molease was employed. PMNs incubated with Str lecular weight) and their ability to inhibit pneumoniae autolysis phase supernatants PMA stimulated superoxide compared with released no more lactate dehydrogenase than that of non-dialysed supernatants. In four exPMNs incubated alone: PMNs alone, $418(92)$ periments the amount of superoxide detected BB units LDH/90 minutes; PMNs + autolysis was as follows: (a) PMNs + PMA 4.71 (0.43); phase supernatant, 402 (83) BB units LDH/90 (b) PMNs + PMA + autolysis supernatant 3.21 minutes; lysed PMNs (total LDH), 1220 (115) BB units $\mathrm{LDH} / 90$ minutes, $\mathrm{n}=7$. (b) PM); (c) PMNs + PMA + dialysed super-
(c.4) natant $3.82(0.34) \mathrm{nmol} \mathrm{O}_{2}^{-} / 10^{5} \quad \mathrm{PMNs} / 90$ 
Table 1 Effect of Streptococcus pneumoniae autolysis phase supernatants on the degranulation response of polymorphonuclear leucocytes (PMNs)

\begin{tabular}{llll}
\hline & $n$ & $\begin{array}{l}\text { Myeloperoxidase released } \\
(\% \text { of total })\end{array}$ & $p$ \\
\hline PMNs + PMA & 7 & $\begin{array}{l}27 \cdot 5 \%(18 \cdot 2 \%) \\
64.0 \%(17.0 \%)\end{array}$ & $<0.05$ \\
PMNs + PMA + supernatant & & $1.7 \%(1.7 \%)$ & NS \\
PMNs alone & 6 & $3.0 \%(2 \cdot 4 \%)$ & \\
PMNs + supernatant & &
\end{tabular}

$\mathrm{PMN}=$ polymorphonuclear leucocyte; $\mathrm{PMA}=$ phorbol myristate acetate.
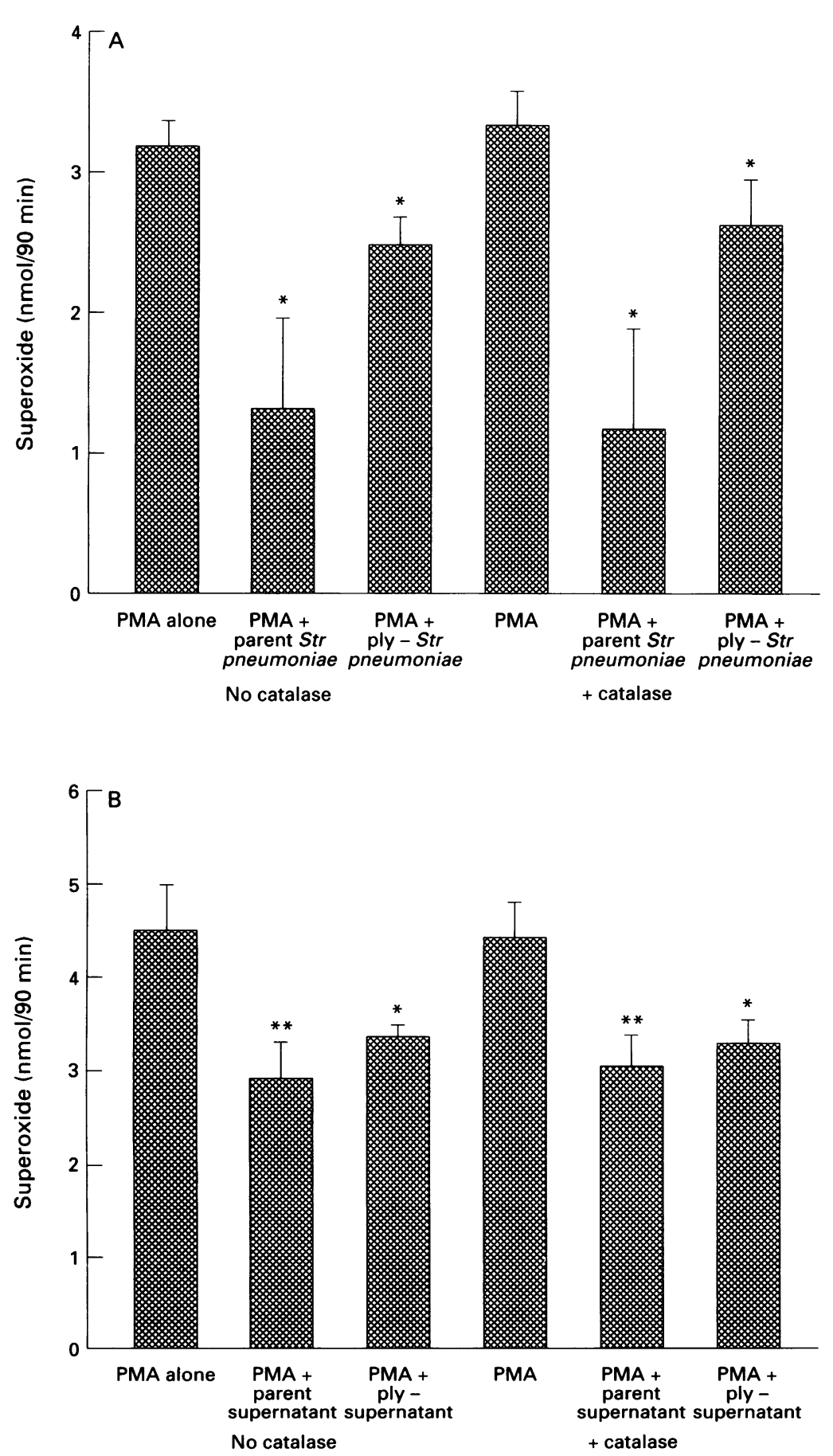

Figure 3 Inhibition of polymorphonuclear leucocyte (PMN) phorbol myristate acetate $(P M A)$-stimulated superoxide production by $(A) \log$ phase parent and pneumolysin-negative (ply-) Str pneumoniae $(n=3)$ and $(B)$ parent and pneumolysin negative Str pneumoniae autolysis phase supernatants $(n=4)$. ${ }_{*} p<0.05,{ }^{* *} p<0.01$ v PMA alone. minutes. Thus, whilst dialysis significantly reduced the inhibitory activity of the supernatant $(\mathrm{p}<0.05, \mathrm{c} v \mathrm{~b})$, dialysed supernatants still possessed significant inhibitory activity $(\mathrm{p}<0.05, \mathrm{c} v \mathrm{a})$. This result suggests that there is a fraction of molecular weight $>10 \mathrm{kD}$ with inhibitory activity and possibly another with molecular weight $<10 \mathrm{kD}$. Since hydrogen peroxide produced by Str pneumoniae can reoxidise cytochrome $c$ and thus interfere with the inhibition assay, ${ }^{1929}$ experiments were set up to determine whether the inhibitory activity of the fractions was sensitive to catalase. The amount of catalase used $(50 \mu \mathrm{g} / \mathrm{ml})$ was that previously shown to completely block reoxidation of reduced cytochrome c by Str pneumoniae. ${ }^{16}$ In the four experiments catalase did not reduce the inhibitory activity of the supernatant: $3.10(0.35)(\mathrm{NS} v \mathrm{~b})$ or the dialysed supernatant: $4 \cdot 15(0 \cdot 17)$ (NS $v \mathrm{c})$. To determine whether inhibitory activity was present in the low as well as the high molecular weight fractions, autolysis phase supernatants were separated through a $10 \mathrm{kD}$ filter and both fractions assessed for inhibitory activity. The results were as follows: (i) PMNs + PMA 4.42 (0.55); (ii) PMNs + PMA + unfractionated supernatant $3.03(0.41)(\mathrm{p}<0.01 \quad v \mathrm{i})$; (iii) PMNs + PMA + > 10 kD fraction 4.01 (0.26) ( $\mathrm{p}<0.05 v$ i); (iv) PMNs + PMA $+<10 \mathrm{kD}$ fraction $3.20(0.49)(\mathrm{p}<0.01 v \mathrm{i}) \mathrm{nmol} \mathrm{O}_{2}{ }^{-} / 10^{5}$ PMNs/90 minutes, $\mathrm{n}=8$, all experiments performed in the presence of $50 \mu \mathrm{g} / \mathrm{ml}$ catalase. These findings confirm the presence of inhibitory activity in both fractions.

ROLE OF PNEUMOLYSIN IN THE INHIBITION OF THE RESPIRATORY BURST OF PMNS BY $S T R$ PNEUMONIAE

To assess the role of pneumolysin in the inhibition two approaches were undertaken. Firstly, the ability of a laboratory mutated strain of Str pneumoniae, which lacks a functional pneumolysin gene, to inhibit the respiratory burst was investigated. Secondly, the effect of antipneumolysin serum on the inhibitory activity of type 1 and type 14 Str pneumoniae was measured.

Ability of pneumolysin-negative Str pneumoniae to inhibit the respiratory burst of PMNs

The ability of log phase pneumolysin negative Str pneumoniae and autolysis phase supernatants to inhibit PMA stimulated superoxide production by PMNs is illustrated in fig 3A. As can be seen, both parent and pneumolysin negative log phase Str pneumoniae significantly inhibited superoxide production by PMNs. The same result was obtained in the presence and absence of catalase. The degree of inhibition between the mutant and parent strain was not significantly different. Supernatants from autolysis phase cultures of both parent and pneumolysin negative Str pneumoniae caused significant inhibition of the PMN respiratory burst (fig 3B) and the same result was obtained in the presence of catalase. Again the dif- 
ference between the extent of inhibition caused by the parent and mutant strains was not significant.

Effect of antipneumolysin serum on the ability of Str pneumoniae to inhibit the respiratory burst of PMNs

Haemolytic assays: before examining the ability of antipneumolysin rabbit serum to reduce the inhibitory effect of Str pneumoniae on PMA stimulated PMN superoxide production it was necessary to determine the ability of the antipneumolysin serum to neutralise pneumolysin. The latter was tested by exploiting the ability of pneumolysin to lyse sheep red blood cells. Purified pneumolysin lysed sheep red blood cells at concentrations at or above $0.3 \mathrm{ng} / \mathrm{ml}$; antipneumolysin serum at $1: 100$ protected sheep red blood cells up to $5 \mathrm{ng} / \mathrm{ml}$ pneumolysin.

Additional experiments were set up to determine whether intact log phase Str pneumoniae and autolysis phase supernatants produced pneumolysin as judged by their capacity to lyse sheep red blood cells. In three experiments log phase type 1 Str pneumoniae caused only slight haemolysis and neither normal rabbit serum nor antipneumolysin serum were protective (Str pneumoniae alone $8.5 \%(1 \cdot 1 \%$ ), Str pneumoniae + normal rabbit serum $7.9 \%$ $(1.3 \%)$, Str pneumoniae + antipneumolysin rabbit serum $6.5 \%(1.9 \%)$ haemolysis). In two of three experiments type 14 Str pneumoniae showed substantial haemolytic activity $(66.4 \%$ and $87.3 \%$ haemolysis respectively). Normal rabbit serum partially protected the sheep red blood cells (28.1\% and $19.9 \%$ haemolysis with normal rabbit serum present) and antipneumolysin rabbit serum protected further $(8.0 \%$ and $8.3 \%$ haemolysis). The third type 14 sample had only a small lytic effect $(9 \cdot 3 \%)$; however, both normal rabbit serum and antipneumolysin rabbit serum completely blocked haemolysis $(0 \%)$.
Ten autolysis supernatants ( $\mathrm{pH}$ adjusted to $7 \cdot 0-7 \cdot 2)$ had very little lytic activity $(2 \cdot 2 \%$ $(2 \cdot 9 \%)$ haemolysis).

Inhibition assays: log phase type 1 and type 14 Str pneumoniae were incubated at room temperature for one hour either alone, with 1:100 normal rabbit serum or with $1: 100$ antipneumolysin rabbit serum. The results are summarised in table 2 . It can be seen that in the absence or presence of catalase neither normal rabbit serum nor antipneumolysin rabbit serum abrogated the inhibitory effect of log phase types 1 or 14 Str pneumoniae.

Table 3 shows the results of similar experiments with autolysis phase supernatants. Again, neither normal rabbit serum nor antipneumolysin rabbit serum had any effect on the inhibition.

\section{Discussion}

We have previously reported that Str pneumoniae inhibits the respiratory burst of PMNs. ${ }^{19}$ The results presented here show that this ability is not shared by two other common lung pathogens, $K$ pneumoniae and Staph aureus. In contrast to Str pneumoniae, these organisms stimulated PMNs to release superoxide. Since reactive oxygen species can cause tissue damage, ${ }^{30}$ including lung injury,,$^{31}$ this observation may help to explain why pneumococcal pneumonia is characterised by a lack of residual lung damage, ${ }^{32}$ whereas tissue necrosis and/ or lung fibrosis are frequently observed in pneumonias caused by $K$ pneumoniae and Staph aureus..$^{32} 33$

These results also indicate that the inhibitory activity of Str pneumoniae is specific for the oxidative response and is not attributable to a non-specific toxic factor. Autolysis phase supernatants which inhibited the respiratory burst did not kill PMNs as assessed by LDH release - confirming our previous observations using trypan blue exclusion - nor did they inhibit the degranulation response of PMNs.

Table 2 Effect of normal rabbit serum (NRS) and antipneumolysin serum (PnIS) on the ability of log phase Streptococcus pneumoniae $(S \mathrm{pn})$ to inhibit phorbol myristate acetate (PMA)-stimulated superoxide production by polymorphonuclear leucocytes (PMNs) (nmol $\mathrm{O}_{2}^{-} / 10^{5} \mathrm{PMNs} / 90$ minutes)

\begin{tabular}{lllll}
\hline & $P M A$ alone & $P M A+S$ pn & PMA $+S$ pn + NRS & PMA $+S$ pn + PnIS \\
\hline Type 1 & & & & \\
$\quad$ No catalase & 3.16 & 1.57 & 1.59 & 1.57 \\
$\quad+50 \mu \mathrm{g} / \mathrm{ml}$ catalase & 3.13 & 2.65 & 2.77 & 2.91 \\
Type 14 & & & & \\
$\quad$ No catalase & 3.16 & 1.70 & 1.57 & 1.52 \\
$\quad+50 \mu \mathrm{g} / \mathrm{ml}$ catalase & 3.13 & 2.59 & 2.91 & 2.69 \\
\hline
\end{tabular}

Results are the mean of two experiments for each serotype.

Table 3 Effect of normal rabbit serum (NRS) and antipneumolysin serum (PnIS) on the ability of autolysis phase supernatants to inhibit phorbol myristate acetate (PMA)-stimulated superoxide production by polymorphonuclear leucocytes $(P M N s)\left(\right.$ nmol $O_{2} / 10^{5} P M N s / 90$ minutes)

\begin{tabular}{lllll}
\hline & $P M A$ alone & PMA supernatant & PMA supernatant + NRS & PMA supernatant + PnIS \\
\hline No catalase & $3.85(0.38)$ & $2.48(0.43)$ & $2.61(0.47)$ & $2.70(0.47)$ \\
$+50 \mu \mathrm{g} / \mathrm{ml}$ catalase & $4.09(0.81)$ & $3.04(0.88)$ & $3.00(0.77)$ & $3.10(0.71)$ \\
\hline
\end{tabular}

Results are mean (SD) of four experiments (two using type 1 and two using type 14 Str pneumoniae).

The inhibitions caused by the supernatants under the different conditions are not significantly different from each other (analysis of variance). 
Rather, the supernatants appeared synergistically to enhance PMA stimulated myeloperoxidase release. It is possible that the enhanced myeloperoxidase release is a consequence of the oxidative response inhibitor since myeloperoxidase is known to be inactivated by reactive oxygen species. ${ }^{34}$

Previous attempts ${ }^{19}$ to determine how Str pneumoniae interferes with the PMN respiratory burst showed that, although the organism produces SOD, the amounts are insufficient to account for the interference observed. Thus, the interference is not due to a scavenging effect of SOD. Here it was shown that washed PMNs which had been preincubated with autolysis phase supernatants exhibited diminished production of hydrogen peroxide upon stimulation with PMA. This result also renders it unlikely that the interference is due to scavenging, but rather that it results from inhibition of the respiratory burst. Again this effect could not be attributed to death of the PMNs as they retained their viability as judged by trypan blue exclusion. The above results also show that the inhibitory effects are demonstrable using different assays for measuring the respiratory burst.

The ability of Str pneumoniae to inhibit the respiratory burst of PMNs may constitute a major counter defence of the microorganism and help to explain its pathogenicity. We therefore sought to characterise the inhibitory factor further. The results indicate that the activity is heat sensitive but that it is not inactivated by acidic conditions. Experiments using dialysed supernatants indicated that a fraction of molecular weight $>10 \mathrm{kD}$ contained inhibitory activity. The loss of some activity on dialysis suggested the possibility of an additional low molecular weight inhibitor which is insensitive to catalase. Experiments using filtered supernatants, in which both the fractions $>10 \mathrm{kD}$ and $<10 \mathrm{kD}$ were retained, confirmed the presence of inhibitory activity in both fractions, in this instance more inhibitory activity being found in the fraction $<10 \mathrm{kD}$.

Some of the attributes of the inhibitory activity we have described are in keeping with the known characteristics of the pneumococcal toxin pneumolysin - for example, pneumolysin is released into the culture supernatant during autolysis, is heat sensitive, and has a molecular weight of approximately $54 \mathrm{kD}$. We therefore investigated whether, or to what extent, pneumolysin was responsible for the inhibition we have reported. The results clearly distinguish our inhibitor from pneumolysin. Firstly, a pneumolysin negative strain of Str pneumoniae was able to inhibit PMA stimulated superoxide production by PMNs. Both log phase organisms and autolysis phase supernatants exerted the inhibitory effect. Secondly, experiments designed to measure the haemolytic activity of Str pneumoniae revealed a dissociation between haemolytic activity and the ability to inhibit the respiratory burst; both types 1 and $14 \log$ phase Str pneumoniae inhibited PMN oxidative responses, ${ }^{19}$ but only type 14 showed substantial haemolytic activity. Furthermore, autolysis phase supernatants were inhibitory despite the fact that they lacked haemolytic activity. A lack of haemolytic activity in itself suggests the absence of active pneumolysin. Such an outcome was not unexpected in the case of the autolysis phase supernatants because those used here were obtained from 18-24 hour cultures and were acidic $(\mathrm{pH} 5.3-$ $6 \cdot 4)$, and it is known that pneumolysin activity declines with prolonged Str pneumoniae culture (beyond 16 hours) due to increasing acidity. ${ }^{28}$ Finally, in confirmation of the conclusion that the inhibitor described here is distinct from pneumolysin, antipneumolysin serum failed to abrogate the inhibitory effect of either $\log$ phase organisms or autolysis phase supernatants from Str pneumoniae despite its efficacy in neutralising pneumolysin in haemolytic assays.

It has been shown that pneumolysin inhibits oxidative responses ${ }^{20}$ Our results suggest that the new inhibitor described here also contributes to the overall inhibitory effect of Str pneumoniae on the respiratory burst. Moreover, since pneumolysin is inactivated by the respiratory burst, ${ }^{35}$ the inhibitor, by interfering with this process, may potentiate the activity of pneumolysin.

Our finding of haemolytic activity in log phase type 14 Str pneumoniae was surprising since extracellular pneumolysin activity has not previously been reported for log phase organisms. This may suggest that, although not released, pneumolysin is exposed on log phase type 14 Str pneumoniae. Alternatively, it is possible that the activity derives from a minority of organisms undergoing autolysis within the population and not from log phase organisms themselves.

In conclusion, these results demonstrate that Str pneumoniae produces a substance other than pneumolysin which inhibits the respiratory burst of PMNs. This substance is biologically active during the logarithmic phase of growth and is manifested by both clinically important and pneumolysin negative strains.

This work was supported by a research grant from the British Lung Foundation. TJM is in receipt of a Royal Society Fellowship.

1 British Thoracic Research Committee. Communityacquired pneumonia in adults in British hospitals in 1982-1983: a survey of aetiology, mortality, prognostic factors and outcome. $Q \mathcal{F}$ Med 1987;62:195-220.

2 Pennington JE. Immunoprophylaxis and immunotherapy of lower respiratory tract infections. In: Pennington JE, ed. Respiratory infections: diagnosis and management. New York: Raven Press, 1983:253-67.

3 Finch $R$. Is pneumococcal infection a preventable disease? f Infect 1988;17:95-8.

4 Ridgway EJ, Allen KD. Penicillin resistance in pneumococci. I Antimicrob Chemother 1991;27:251-2.

Klugman KP. Worldwide increase in pneumococcal antibiotic resistance. Lancet 1989;ii:444.

6 Casal J, Fenoll A, Vicioso MD, Munoz R. Increase in resistance to penicillin in pneumococci in Spain. Lancet 1989;i:735.

7 Pallett AP, Strangeways JEM. Penicillin-resistant pneumococci. Lancet 1988;i:1452.

8 Shapiro ED, Berg AT, Austrian R, Schroeder D, Parcells $\mathrm{V}$, Margolis A. The protective efficacy of polyvalent pneumococcal polysaccharide vaccine. $N$ Engl $\boldsymbol{f}$ Med 1991;325:1453-60.

9 Fine DP, Kirk JL, Schiffman G, Schweinle JE, Guckian JC. Analysis of humoral and phagocytic defences against Streptococcus pneumoniae serotypes 1 and 2 . $\mathcal{~ L a b ~ C l i n}$ Med 1988;112:487-97. 
10 Gillespie SH. Aspects of pneumococcal infection including bacterial virulence, host response and vaccination. $f \mathrm{Med}$ Microbiol 1989;28:237-48.

11 Wilson CB, Tasi V, Remington JS. Failure to trigger the oxidative metabolic burst by normal macrophages. $\mathcal{f}$ Exp Med 1980;151:328-46.

12 Murray HW, Juangbhanich CW, Nathan CF, Cohn Z. Macrophage oxygen-dependent antimicrobial activity.
7 Exp Med 1979;150:950-64.

13 Johnston RB, Keele BB, Misra HP, Lehmeyer JE, Webb LS, Baehner RL. The role of superoxide anion generation in phagocytic bactericidal activity. $f$ Clin Invest 1975;55:1357-72.

14 Braconier JH, Odeberg G. Granulocyte phagocytosis and killing of virulent and avirulent serotypes of Streptococcus pneumoniae. $\mathcal{F}$ Lab Clin Med 1982;100:279-87.

15 Perry FE, Elson CJ, Catterall JR. Susceptibility of $S$ pneumoniae to killing by reactive oxygen species. Thorax 1992;47:869.

16 Thore M, Lofgren S, Tarnvik A, Monsen T, Selstam E, Burman LG. Anaerobic phagocytosis, killing, and degradation of Streptococcus pneumoniae by human peripheral dation of Streptococcus pneumoniae by human pe
blood leukocytes. Infect Immun 1985;47:277-81.

17 Esposito AL, Clark CA, Poirier WJ. An assessment of the factors that contribute to the killing of type 3 Streptococcus pneumoniae by human peripheral blood leukocytes in vitro. APMIS 1990;98:111-21.

18 Perry FE, Elson CJ, Catterall JR. Stimulation of the oxidative response of neutrophils by cell associated Streptococcus pneumoniae. Thorax 1992;47:870.

19 Perry FE, Elson CJ, Greenham LW, Catterall JR. Interference of the oxidative response of neutrophils by Streptococcus pneumoniae Thorax 1993;48:364-9.

20 Paton JC, Ferrante A. Inhibition of human polymorphonuclear leukocyte respiratory burst, bactericidal activity and migration by pneumolysin. Infect Immun and migration

21 Berry AM, Yother J, Briles DE, Hansman D, Paton DC. Reduced virulence of a defined pneumolysin-negative mutant of Strep

22 Miles AA, Misra SS. The estimation of the bacterial power of the blood. $\mathcal{F}$ Hyg 1938;38:732-9.

23 Dooley DC, Simpson JF, Meryman HT. Isolation of large numbers of fully viable human neutrophils: a preparative technique using percoll density gradient centrifugation. Exp Haematol 1982;10:591-9.

24 Pick E, Mizel D. Rapid microassays for the measurement of superoxide and hydrogen peroxide production by macrophages in culture using an automatic enzyme immunoassay reader. $\mathcal{F}$ Immunol Methods 1981;46:211-26.

25 Suzuki K, Ota H, Sasagawa S, Sakatani T, Fujikura T. Assay method for myeloperoxidase in human polymorphonuclear leukocytes. Anal Biochem 1983;132:345-52.

26 Paton JC, Lock RA, Lee C-J, Li JP, Barry AM, Mitchell $\mathrm{TJ}$, et al. Purification and immunogenicity of genetically obtained pneumolysin toxoids and their conjugation to Streptococcus pneumoniae type $19 \mathrm{~F}$ polysaccharide. Infect Immun 1991;59:2297-304.

27 Mitchell TJ, Walker FK, Saunders FK, Andrew PW, Boulnois GJ. Expression of the pneumolysin gene in Escherichia coli: rapid purification and biological properties. Biochem Biophys Acta 1989;1007:67-72.

28 Steinfort C, Wilson R, Mitchell T, Feldman C, Rutman A, Todd $\mathrm{H}$, et al. Effect of Streptococcus pneumoniae on human respiratory epithelium in vitro. Infect Immun human respiratory

29 Vanderwalle PL, Petersen NO. Oxidation of reduced cytochrome c by hydrogen peroxide. FEBS Lett

30 Weiss SJ. Tissue destruction by neutrophils. $N$ Engl f Med 1989;320:365-76.

31 Johnson KJ, Fantone JC, Kaplan J, Ward PA. In vivo damage of rat lungs by oxgygen metabolites. $\mathcal{f}$ Clin Invest 1981;67:983-93.

32 Farr BM, Mandell GL. Gram-positive pneumonia. In: Pennington JE, ed. Respiratory infections: diagnosis and management. 2nd edn. New York: Raven Press, 1989:302.

33 Crane LR, Komshian S. Gram-negative bacillary pneumonias. In: Pennington JE, ed. Respiratory infections: diagnosis and management. 2nd edn. New York: Raven Press, 1989:321.

34 Edwards SW, Nurcombe HL, Hart CA. Oxidative inactivation of myeloperoxidase released from human neutrotion of myeloperoxidase released

35 Clark RA. Oxidative inactivation on pneumolysin by the myeloperoxidase system and stimulated human neutrophils. F Immunol 1986;136:4617-22. 